\title{
Quantitative determination of domain wall coupling energetics
}

\author{
M. Laufenberg, D. Bedau, H. Ehrke, M. Kläui, ${ }^{a)}$ and U. Rüdiger \\ Fachbereich Physik, Universität Konstanz, Universitätsstraße 10, 78457 Konstanz, Germany \\ D. Backes, ${ }^{\text {b) }}$ L. J. Heyderman, and F. Nolting \\ Paul Scherrer Institut, 5232 Villigen PSI, Switzerland \\ C. A. F. Vaz and J. A. C. Bland \\ Cavendish Laboratory, University of Cambridge, Madingley Road, Cambridge CB3 OHE, United Kingdom \\ T. Kasama and R. E. Dunin-Borkowski \\ Frontier Research System, The Institute of Physical and Chemical Research, Hatoyama, \\ Saitama 350-0395, Japan and Department of Materials Science and Metallurgy, University of Cambridge, \\ Pembroke Street, Cambridge CB2 3QZ, United Kingdom
}

S. Cherifi, A. Locatelli, and S. Heun ${ }^{\text {) }}$

Sincrotrone Trieste, 34012 Basovizza, Trieste, Italy

(Received 13 May 2005; accepted 26 April 2006; published online 26 May 2006)

\begin{abstract}
The magnetic dipolar coupling of head-to-head domain walls is studied in $350 \mathrm{~nm}$ wide $\mathrm{NiFe}$ and Co nanostructures by high resolution magnetic imaging. We map the stray field of a domain wall directly with sub-10-nm resolution using off-axis electron holography and find that the field intensity decreases as $1 / r$ with distance. By using x-ray magnetic circular dichroism photoemission electron microscopy, we observe that the spin structures of interacting domain walls change from vortex to transverse walls, when the distance between the walls is reduced to below $(77 \pm 5) \mathrm{nm}$ for $27 \mathrm{~nm}$ thick NiFe and $(224 \pm 65) \mathrm{nm}$ for $30 \mathrm{~nm}$ thick Co elements. Using measured stray field values, the energy barrier height distribution for the nucleation of a vortex core is obtained. (C) 2006 American Institute of Physics. [DOI: 10.1063/1.2207220]
\end{abstract}

Ferromagnetic nanostructures with geometrically confined domain walls have been in the focus of increasing interest recently because of possible applications in different fields of nanotechnology such as nonvolatile magnetic random access memory ${ }^{1}$ (MRAM) and magnetic logic based on domain walls. ${ }^{2}$ Magnetic switching by current-induced domain wall motion has recently initiated a new exciting field of research based on the interaction between spin currents and domain walls. ${ }^{3}$ The spin structures of head-to-head or tail-to-tail $180^{\circ}$ domain walls have been studied previously for noninteracting isolated walls. ${ }^{4}$ Two types of walls are formed, transverse or vortex walls depending on the width, thickness, and material. ${ }^{5-7}$

A strong influence of the domain wall interaction on the switching of magnetic elements such as rings was found recently, when interaction-induced collective switching of adjacent elements was observed for small spacings. 8 Such switching is dominated by domain wall motion and can only be understood with a detailed knowledge of the interacting domain walls' spin structures. Theoretically, the energies of the two domain wall types are different when interacting with an external field. We therefore expect the dipolar coupling to affect the two wall types in different ways and coupling-induced transitions from one domain wall type to another may occur. ${ }^{5}$ A deeper understanding of the energetics involved in such interactions is only possible if the stray fields of domain walls are determined quantitatively. This

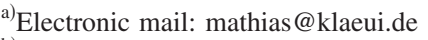

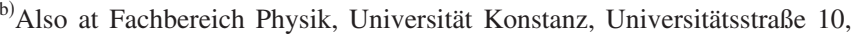
78457 Konstanz, Germany.

${ }^{c}$ Present address: TASC-INFM Laboratory, Area Science Park, 34012 Ba-
} sovizza, Trieste, Italy. knowledge can then provide a basis for understanding the interaction-dependent domain wall spin structure and the resulting magnetic behavior.

In this letter, we report the results of a quantitative study of the domain wall coupling, including the critical distances at which the interactions become significant, by the direct high resolution $(<10 \mathrm{~nm})$ measurement of domain wall stray fields using off-axis electron holography. Using x-ray magnetic circular dichroism photoemission electron microscopy (XMCD-PEEM) a spacing-dependent domain wall transition from transverse to vortex walls is detected. Our measurements provide a direct measure of the energy barrier distribution for the nucleation of vortex cores in these structures.

Arrays of $5 \times 5$ rings, each of outer diameter of $1.64 \mu \mathrm{m}$ and width of $350 \mathrm{~nm}$, were fabricated by electron beam lithography, molecular beam epitaxy of polycrystalline NiFe and $\mathrm{Co}$, and lift-off as described in Ref. 10. Edge-to-edge spacings between rings down to $10 \mathrm{~nm}$ were used to investigate different dipolar coupling strengths between domain walls in adjacent rings. For the transmission off-axis electron holography experiments, 3/4 rings were patterned from $27 \mathrm{~nm}$ thick Co films on $50 \mathrm{~nm}$ thick SiN membranes. ${ }^{11}$ Open rings were patterned on the fragile membranes in order to facilitate the lift-off process which cannot be assisted by ultrasound, in contrast to the PEEM samples grown on naturally oxidized Si substrates. PEEM images of domain walls in 3/4 rings and full rings with the same dimensions showed no difference in the domain wall spin structure.

The nonintrusive technique of XMCD-PEEM ${ }^{12}$ was used to image the spin structure of the domain walls in the rings. The stray field of the walls was quantitatively determined using off-axis electron holography. ${ }^{13}$ 


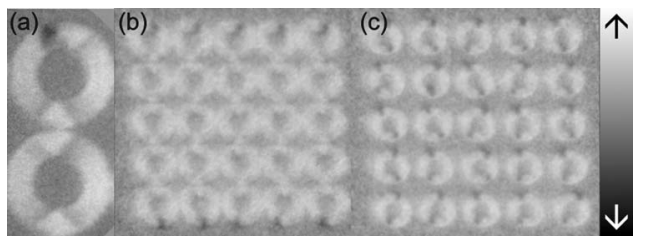

FIG. 1. (a) High resolution XMCD-PEEM image of two rings in the onion state, after saturation with an external field in the vertical direction and relaxation. White and black contrasts correspond to the magnetization pointing up and down, respectively. A noninteracting vortex wall (top) and three interacting transverse walls are visible. Overview images of an array of $27 \mathrm{~nm}$ thick and $350 \mathrm{~nm}$ wide NiFe rings with an edge-to-edge spacing of (b) $40 \mathrm{~nm}$ and (c) $500 \mathrm{~nm}$, respectively. Since domain walls at the top and bottom edges of the array do not interact with adjacent walls, they are vortex walls for all spacings investigated.

After saturating with a magnetic field and relaxing the field, rings attain the onion state characterized by two headto-head domain walls [Fig. 1(a)]. An array of 25 rings in the onion state exhibits 50 walls in total. The domain walls inside the array interact with adjacent walls via their stray fields. Ten walls, which are located at the two opposite edges [top and bottom edges in Figs. 1(b) and 1(c)] of the array are not influenced by stray fields of an adjacent wall. For all experiments, ring thickness and width were chosen such that isolated rings of this geometry exhibit vortex walls. ${ }^{6,7}$ Figure 1 shows XMCD-PEEM images of arrays of $27 \mathrm{~nm}$ thick NiFe rings with (b) $40 \mathrm{~nm}$ and (c) $500 \mathrm{~nm}$ edge-to-edge spacings, respectively, as well as a high resolution image (a) showing both wall types. Vortex walls can be easily identified by black and white contrast which occurs because all magnetization directions corresponding to the full gray scale are present in a vortex. In contrast, transverse walls exhibit the characteristic gray-white-gray contrast of the triangular spin structure. In Fig. 2, we show the percentage of transverse walls inside the array as function of the edge-to-edge spacing for $27 \mathrm{~nm}$ thick NiFe rings (black squares) extracted from images of the type shown in Fig. 1. A decreasing number of transverse walls is found with increasing spacing. Domain walls at the edges of the arrays are vortex walls irrespective of the spacing due to the absence of dipolar coupling with adjacent walls. The data points for infinite spacings in Fig. 2 result from these domain walls. The transverse to vortex transition is characterized by a (10\%-90\%) width of the switching distribution of $w=(65 \pm 9) \mathrm{nm}$ and a

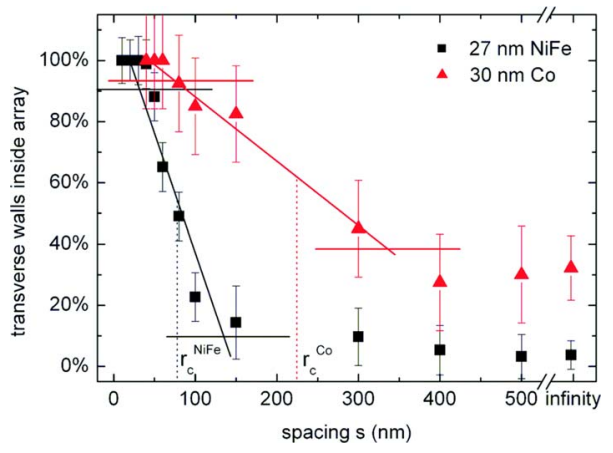

FIG. 2. (Color online) Percentage of transverse walls inside a ring array as function of edge-to-edge spacing for $27 \mathrm{~nm} \mathrm{NiFe}$ (black squares) and $30 \mathrm{~nm}$ Co (red triangles). The error bars represent the absolute statistical error $1 / \sqrt{n}$ due to the finite number $n$ of domain walls investigated. The horizontal lines show the 10\%-90\% levels of the transition from a transverse to a vortex domain wall from which the width $w$ and the center $r$ are extracted. center at $r_{c}=(77 \pm 5) \mathrm{nm}$. In Fig. 2 , red triangles show a similar transition for $30 \mathrm{~nm}$ thick Co rings with $w$ $=(328 \pm 130) \mathrm{nm}$ and $r_{c}=(224 \pm 65) \mathrm{nm}$.

In order to explain these results, we first consider the process of domain wall formation in an isolated ring. When relaxing the applied external field from saturation, transverse walls are initially formed. In order to create a vortex wall, a vortex core has to be nucleated. This hysteretic transition from one wall type to the other involves overcoming a local energy barrier, ${ }^{14}$ since the nucleation of the vortex core is associated with a strong twisting of the spins in the core region. ${ }^{15}$ In arrays of interacting rings, the edge-to-edge spacing-dependent stray field stabilizes transverse walls so that for small spacings (corresponding to a strong stray field from the adjacent domain wall) transverse walls are favored [Fig. 1(b)]. For increasing spacing, the influence of the stray field from an adjacent wall is reduced, until vortex walls are formed in the rings with the lowest energy barrier for the vortex core nucleation. The further the spacing increases the more rings nucleate vortex walls [Fig. 1(c)]. Thus the spacing at which a wall switches from transverse to vortex is related to the nucleation barrier, which depends on local imperfections such as the edge roughness. Therefore the number of domain walls that has switched from transverse to vortex as a function of the edge-to-edge spacing is a measure of the distribution of energy barriers for the vortex core nucleation. For NiFe, a relatively sharp transition occurs from all walls being transverse to all walls being vortex walls, while for Co rings the transition is much wider. This difference is thought to result from the different polycrystalline microstructures of the NiFe (magnetically soft fcc crystallites with negligible anisotropy) and the Co (hcp crystallites with strong uniaxial anisotropy leading to a larger number of pinning sites). Furthermore, this results in the presence of transverse walls in our Co sample even at infinite spacings. Thus Co had to be chosen for the electron holography measurements rather than $\mathrm{NiFe}$ in order to be able to observe a transverse wall in an isolated structure at all.

This spacing-dependent distribution for the vortex core nucleation needs to be transformed to a distribution as a function of the stray field, which is as a first approximation proportional to the energy. To do this, the stray field as well as the magnetization of the domain wall was imaged using off-axis electron holography. The inset of Fig. 3 shows an image of the in-plane magnetic induction integrated in the electron beam direction, obtained from a transverse wall in a $27 \mathrm{~nm}$ thick isolated Co 3/4 ring designed with the same width as that of the rings imaged by XMCD-PEEM. No significant difference between the functional dependence of the stray field on the spacing is expected for a 27 and a $30 \mathrm{~nm}$ thick sample. The stray field was measured along the length of the region indicated in the image, and is shown as a function of the distance $r$ from the ring edge in Fig. 3, normalized to the saturation magnetization of Co. The line is a $1 / r$ fit which can be expected for the distance dependence of the stray field created by an area of magnetic poles for small $r{ }^{16}$ This dependence also confirms earlier results from indirect Kerr effect measurements. ${ }^{9}$ In order to obtain the stray field of one single domain wall acting on an adjacent wall, the stray field of an isolated wall was imaged.

The spacing-dependent energy barrier distribution is now rescaled to a field-dependent distribution using the measured stray field decay of Fig. 3 and presented in Fig. 4. The 


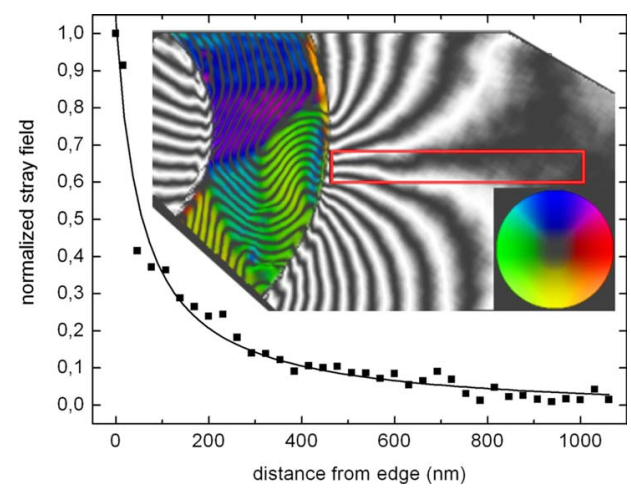

FIG. 3. (Color online) The inset shows an off-axis electron holography image of a transverse wall in a $27 \mathrm{~nm}$ thick Co $3 / 4$ ring. The color code indicates the direction of the in-plane magnetization and the black lines represent directly the stray field. The stray field strength was measured at several distances inside the marked area. The data points show the stray field normalized to the saturation magnetization as a function of the distance $r$ from the ring edge for the wall shown in the inset. The line is a $1 / r$ fit.

rescaled data can be fitted with the error function $\operatorname{erf}(x)$, which is the integral of a Gaussian distribution. ${ }^{16}$ This suggests a Gaussian distribution for the energy barriers in agreement with the presence of independent local pinning centers at the particular wall position that determine the nucleation barrier. The position of the maximum is $H_{\max } / M_{s}$ $=0.21 \pm 0.10$ and the full width at half maximum $w / M_{s}$ $=0.16 \pm 0.05$, where $M_{s}$ is the saturation magnetization. Using $E_{\max }=\frac{1}{2} \mu_{0} M_{s} H_{\max }$, an energy density of $E_{\max }$ $=(8.4 \pm 4.0) \times 10^{4} \mathrm{~J} / \mathrm{m}^{3}$ equivalent to the field $H_{\max }$ can be obtained for the $30 \mathrm{~nm}$ thick Co sample.

Figure 2 shows that the transition for the Co sample saturates at a finite value for large spacings. In terms of the model described above, this means that an additional effective field would be needed to overcome the pinning of the remaining transverse walls at structural imperfections and to allow the vortex core nucleation. Since the pinning is much stronger in our Co sample than in the NiFe sample, this occurs here only for Co.

In conclusion, the stray field coupling of head-to-head domain walls in $\mathrm{NiFe}$ and Co nanostructures has been studied by high spatial resolution magnetic imaging. We have mapped the stray field of a domain wall directly using offaxis electron holography with sub-10-nm resolution. We find that the field strength falls off with a $1 / r$ dependence. For

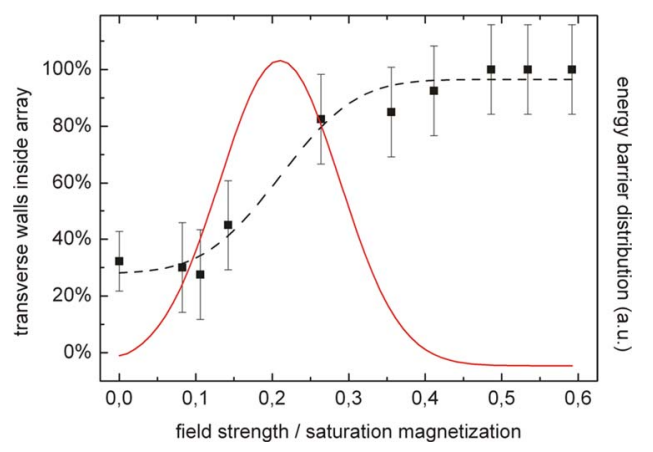

FIG. 4. (Color online) The black squares are the same data points as presented in Fig. 2 for the $30 \mathrm{~nm}$ thick Co sample, but as a function of the normalized field strength. The black dashed line shows a fit with the error function. The corresponding Gaussian distribution of the energy barriers is shown as a full red line. interacting domain walls in $350 \mathrm{~nm}$ wide ring structures, we observe a transition from a transverse to a vortex spin structure with increasing edge-to-edge spacing. For walls in $27 \mathrm{~nm}$ thick NiFe structures, we find a narrow distribution with a width of $w=(65 \pm 9) \mathrm{nm}$ at $r_{c}=(77 \pm 5) \mathrm{nm}$. Due to the larger stray field of the $30 \mathrm{~nm}$ thick Co walls, here $r_{c}$ equals $(224 \pm 65) \mathrm{nm}$ with a broader distribution width of $w$ $=(328 \pm 130) \mathrm{nm}$ than in the soft NiFe. By correlating this distribution with the measured stray field, we obtain the energy barrier distribution for vortex core nucleation. This distribution has a Gaussian profile with $H_{\max } / M_{s}=0.21 \pm 0.10$ and $w / M_{s}=0.16 \pm 0.05$ and the field at the maximum is equivalent to an energy density of $(8.4 \pm 4.0) \times 10^{4} \mathrm{~J} / \mathrm{m}^{3}$.

The authors acknowledge support by the DFG (SFB 513) and by the European Commission through the 6th Framework Programme (contract RII3-CT-2004-506008). One of the authors (D.B.) acknowledges support by the European Union through the European Regional Development Fund (Interreg III A program). Another author (R.E. D.-B.) acknowledges support by the Royal Society. Part of this work was performed at the Swiss Light Source, Paul Scherrer Institute, Villigen, Switzerland [Figs. 1(b) and 1(c), data for Fig. 2] and at Elettra, Trieste, Italy [Fig. 1(a), data for Fig. 2].

${ }^{1}$ G. A. Prinz, J. Magn. Magn. Mater. 200, 57 (1999).

${ }^{2}$ D. A. Allwood, G. Xiong, M. D. Cooke, C. C. Faulkner, D. Atkinson, N. Vernier, and R. P. Cowburn, Science 296, 2003 (2002).

${ }^{3}$ A. Yamaguchi, T. Ono, S. Nasu, K. Miyake, K. Mibu, and T. Shinjo, Phys. Rev. Lett. 92, 077205 (2004); M. Kläui, C. A. F. Vaz, J. A. C. Bland, W. Wernsdorfer, G. Faini, E. Cambril, L. J. Heyderman, F. Nolting, and U. Rüdiger, ibid. 94, 106601 (2005).

${ }^{4}$ M. Kläui, C. A. F. Vaz, L. Lopez-Diaz, and J. A. C. Bland, J. Phys.: Condens. Matter 15, R985 (2003).

${ }^{5}$ R. D. McMichael and M. J. Donahue, IEEE Trans. Magn. 33, 4167 (1997).

${ }^{6}$ M. Kläui, C. A. F. Vaz, J. A. C. Bland, L. J. Heyderman, F. Nolting, A. Pavlovska, E. Bauer, S. Cherifi, S. Heun, and A. Locatelli, Appl. Phys. Lett. 85, 5637 (2004).

${ }^{7}$ M. Laufenberg, D. Backes, W. Bührer, D. Bedau, M. Kläui, U. Rüdiger, C. A. F. Vaz, J. A. C. Bland, L. J. Heyderman, F. Nolting, S. Cherifi, A. Locatelli, R. Belkhou, S. Heun, and E. Bauer, Appl. Phys. Lett. 88, 052507(2006).

${ }^{8}$ X. Zhu, P. Grütter, V. Metlushko, Y. Hao, F. J. Castano, C. A. Ross, B. Ilic, and H. I. Smith, J. Appl. Phys. 93, 8540 (2003).

${ }^{9}$ M. Kläui, C. A. F. Vaz, J. A. C. Bland, and L. J. Heyderman, Appl. Phys. Lett. 86, 032504 (2005)

${ }^{10}$ L. J. Heyderman, C. David, M. Kläui, C. A. F. Vaz, and J. A. C. Bland, J. Appl. Phys. 93, 10011 (2003);Y. G. Yoo, M. Kläui, C. A. F. Vaz, L. J. Heyderman, and J. A. C. Bland, Appl. Phys. Lett. 82, 2470 (2003).

${ }^{11}$ L. J. Heyderman, M. Kläui, R. Schäublin, U. Rüdiger, C. A. F. Vaz, J. A. C. Bland, and C. David, J. Magn. Magn. Mater. 290-291, 86 (2005).

${ }^{12}$ J. Stöhr, Y. Wu, B. D. Hermsmeier, M. G. Samant, G. R. Harp, S. Koranda, D. Dunham, and B. P. Tonner, Science 259, 658 (1993); J. Stöhr, H. A. Padmore, S. Anders, T. Stammler, and M. R. Scheinfein, Surf. Rev. Lett. 5, 1297 (1998).

${ }^{13}$ A. Tonomura, Adv. Phys. 41, 59 (1992); R. E. Dunin-Borkowski, M. R. McCartney, B. Kardynal, S. S. P. Parkin, M. R. Scheinfein, and D. J. Smith, J. Microsc. 200, 187 (2000).

${ }^{14}$ M. Kläui, C. A. F. Vaz, W. Wernsdorfer, E. Bauer, S. Cherifi, S. Heun, A. Locatelli, G. Faini, E. Cambril, L. J. Heyderman, and J. A. C. Bland, Physica B 343, 343 (2004).

${ }^{15}$ R. P. Cowburn, D. K. Koltsov, A. O. Adeyeye, M. E. Welland, and D. M. Tricker, Phys. Rev. Lett. 83, 1042 (1999).

${ }^{16}$ M. McCaig, Permanent Magnets in Theory and Practice, 1st ed. (Pentech, London, 1977).

${ }^{17}$ Assuming a similar dependence of the stray field for $\mathrm{NiFe}$, a Gaussian distribution is found for the energy barrier distribution for $\mathrm{NiFe}$ as well. 